\title{
Simulation of a closed low-pressure honeycomb adsorber for thermal energy storage
}

\author{
M. Schaefer ${ }^{\mathrm{a}, *}$, A. Thess ${ }^{\mathrm{a}, \mathrm{b}}$ \\ ${ }^{a}$ Institute of Energy Storage, University of Stuttgart, Pfaffenwaldring 31, 70569 Stuttgart, Germany \\ ${ }^{b}$ Institute of Engineering Thermodynamics, DLR - German Aerospace Center, Pfaffenwaldring 38-40, 70569 Stuttgart, Germany
}

\begin{abstract}
The efficient implementation of renewable energy sources necessitates thermal energy storages. For domestic as well as industrial applications thermal energy storages based on closed adsorption are studied. Against this background, a closed low-pressure honeycomb adsorber is numerically examined in this work. The examined adsorber contains stacked layers of honeycomb blocks with rectangular channels which are separated by heat exchanger plates. Zeolite $13 \mathrm{X}$ and water is assumed as the adsorption pair. The focus of this work is solely on the adsorption process. The numerical model applies an one-dimensional model for the single channels of the honeycomb blocks. The one-dimensional model has been presented in a previous work of the authors. To account for transversal heat conduction in the honeycomb cross-section, the one-dimensional model equations are extended by heat source/sink terms. In addition, the mass transport equation is modified for rectangular channel flow. The results demonstrate that the heat and mass transfer and the adsorption processes are strongly coupled and can be only understood by their interaction. Regarding modelling aspects, it is found that the spatial variations of temperature and pressure as well as the local deviation from adsorption equilibrium are significant. Hence, no equilibrium assumptions should be made. Further, the minor rarefaction effect of slip should be considered. With respect to the application, the analysis yields that the thermal power can be optimized by variation of the honeycomb geometry parameters, e. g. channel size. The local optimum is a result of the inverse dependencies of the external and internal mass transfer resistance on the channel size. Interestingly, the optimum for peak and mean power do not coincide in general. Finally, it is found that the thermal power can be controlled effectively by the inlet pressure.
\end{abstract}

Keywords:

Adsorption, Zeolite, Honeycomb, Vacuum, Thermal Energy Storage, Simulation

\section{Introduction}

\subsection{Motivation and subject matter}

The efficient implementation of renewable energy sources necessitates both electrical and thermal energy storages (TES). For domestic as well as industrial applications, TESs based on closed adsorption are studied, e.g. [1,2]. To date most publications focus on packed-bed adsorbers with adsorbent pellets or powder filling. More recently, structured honeycomb adsorbents have been developed to improve the storage performance by reducing the heat and mass transfer resistance, e.g. [3, 4]. Here, in accordance with literature, the term 'honeycomb adsorbent' refers to all extruded adsorbents independent of the shape of the channels' cross-section, e.g. [5, 6]. Against this background, a closed low-pressure honeycomb adsorber is numerically examined in this work.

The one-dimensional model for a single channel of the honeycomb adsorbent has been presented and discussed for a basic adsorber set-up in [7]. Here, this model is applied and modified to simulate a more practical adsorber set-up, see Fig. 1. The insulated adsorber contains stacked layers of honeycomb blocks with rectangular channels which are separated by heat

\footnotetext{
${ }^{*}$ Corresponding author

Email address: schaefer@ies. uni-stuttgart.de (M. Schaefer)
}

exchanger plates. In general, this set-up enhances the heat transfer between the adsorbent and the heat exchanger, thus, improving the storage performance in terms of charging and discharging duration and power. As the adsorption pair of zeolite $13 \mathrm{X}$ and water is often studied in applied research, e. g. [8, 9], this pair is also assumed in this study. With water as adsorbate, the in- and outlet pressure of the adsorber typically lies in the range of $p_{\text {in }} \approx 10 \ldots 100 \mathrm{mbar}$, compare e.g. [10].Finally, the focus of this work is solely on the adsorption, that is the discharging process.

\subsection{Literature review}

Regarding the simulation of closed low-pressure adsorbers, most publications focus on packed-bed adsorbers filled with spherical adsorbent particles [11, 12, 13, 14] or on adsorbers with coated heat exchanger tubes, e.g. [15]. Furthermore, the field of application covers mostly heat pumps and only few publications deal with TES, e.g. [16]. Hence, the adsorbers analysed in most publications are of small scale compared to the adsorbers examined in this work and thus the limitation of the TES performance by the heat and mass transfer might be more significant.

The application of honeycomb adsorbers for TES is studied in $[3,4,6]$. The focus lies on the description of the manufacturing process of the honeycomb adsorbents and the physical 


\begin{tabular}{|c|c|c|c|}
\hline \multicolumn{4}{|c|}{ Nomenclature } \\
\hline & & $T_{\mathrm{htx}}$ & temperature of heat exchanger $(\mathrm{K})$ \\
\hline$a$ & honeycomb channel height $(\mathrm{m})$ & $T_{\mathrm{s}}$ & saturation temperature $(\mathrm{K})$ \\
\hline$A$ & cross-section area $\left(\mathrm{m}^{2}\right)$ & $u$ & mean vapour velocity in honeycomb channel $(\mathrm{m} / \mathrm{s})$ \\
\hline$b$ & honeycomb channel width $(\mathrm{m})$ & $W$ & honeycomb width (m) \\
\hline$c$ & specific heat capacity at constant volume (J/kg K) & $X$ & water uptake of zeolite $(\mathrm{kg} / \mathrm{kg})$ \\
\hline$c_{p}$ & specific heat capacity at constant pressure $(\mathrm{J} / \mathrm{kg} \mathrm{K})$ & $X_{\text {eq }}$ & water uptake at adsorption equilibrium $(\mathrm{kg} / \mathrm{kg})$ \\
\hline$f$ & honeycomb web thickness (half) (m) & $x, y, z$ & cartesian coordinates of honeycomb block (m) \\
\hline$G_{\mathrm{P}}$ & Poiseuille coefficient (-) & $\Delta x, \Delta y$ & $\Delta z$ knot spacing of discretization (m) \\
\hline$G_{\mathrm{T}}$ & thermal creep coefficient (-) & Greek & ymbols \\
\hline$\Delta h_{\mathrm{a}}$ & heat of adsorption $(\mathrm{J} / \mathrm{kg})$ & $\gamma_{a}, \gamma_{b}$ & aspect ratio of outer size of honeycomb channel cut- \\
\hline$i$ & honeycomb channel index $(-)$ & & out to channel size $(-)$ \\
\hline$k_{\mathrm{a}}$ & adsorption kinetics parameter $\left(\mathrm{s}^{-1}\right)$ & $\Gamma\left(G_{\mathrm{P}}\right)$ & relative error of the non-dimensional mass flow) \\
\hline$K_{\mathrm{I}, \mathrm{i}}$ & thermal power controller parameters & $\delta$ & local rarefaction parameter $(-)$ \\
\hline Kn & Knudsen number $(-)$ & $\varepsilon$ & honeycomb porosity, zeolite micro-porosity (-) \\
\hline$l_{\mathrm{mol}}$ & mean free path of vapour molecules (m) & $\zeta_{\mathrm{i}}$ & parameters of slip approach of $G_{\mathrm{P}}$-function A.2 (-) \\
\hline$L$ & honeycomb length $(\mathrm{m})$ & $\lambda$ & heat conductivity $(\mathrm{W} / \mathrm{m} \mathrm{K})$ \\
\hline$m$ & mass $(\mathrm{kg})$ & $\lambda_{\mathrm{eff}}$ & effective heat conductivity (W/m K) \\
\hline$\dot{m}$ & vapour mass flow rate $(\mathrm{kg} / \mathrm{s})$ & $\xi_{\mathrm{i}}$ & fitting parameters of $G_{\mathrm{T}}-$ function A.3 (-) \\
\hline$N_{\mathrm{c}}$ & $\begin{array}{l}\text { number of channels in one column or row of a hon- } \\
\text { eycomb block (-) }\end{array}$ & & $\begin{array}{l}\text { density }\left(\mathrm{kg} / \mathrm{m}^{3}\right) \\
\text { volumetric heat source } / \text { sink term }\left(\mathrm{W} / \mathrm{m}^{3}\right)\end{array}$ \\
\hline$p$ & vapour pressure $(\mathrm{Pa})$ & \multicolumn{2}{|c|}{ Subscripts } \\
\hline$p_{\text {th }}$ & volumetric thermal power $\left(\mathrm{W} / \mathrm{m}^{3}\right)$ & 0 & initial state \\
\hline$p_{\text {th,mean }}$ & volumetric mean thermal power $\left(\mathrm{W} / \mathrm{m}^{3}\right)$ & $\mathrm{a}$ & adsorbate, adsorption \\
\hline$p_{\text {th,peak }}$ & volumetric peak thermal power $\left(\mathrm{W} / \mathrm{m}^{3}\right)$ & c & channel \\
\hline$p_{\text {th,set }}$ & $\begin{array}{l}\text { controller set point value of volumetric thermal } \\
\text { power }\left(\mathrm{W} / \mathrm{m}^{3}\right)\end{array}$ & $\begin{array}{l}\text { in } \\
\max \end{array}$ & $\begin{array}{l}\text { inlet of adsorber } \\
\text { maximum }\end{array}$ \\
\hline$R_{\mathrm{S}}$ & specific gas constant of vapour $(\mathrm{J} / \mathrm{kg} \mathrm{K})$ & & reference state \\
\hline$t$ & time $(\mathrm{s})$ & & vapour \\
\hline & non-dimensional time $\tilde{t}:=t / t_{\text {tot }}(-)$ & & zeolite \\
\hline$t_{\mathrm{tot}}$ & total process duration $(\mathrm{s})$ & \multicolumn{2}{|c|}{ Abbreviations } \\
\hline$T$ & temperature $(\mathrm{K})$ & TES & thermal energy storage \\
\hline
\end{tabular}

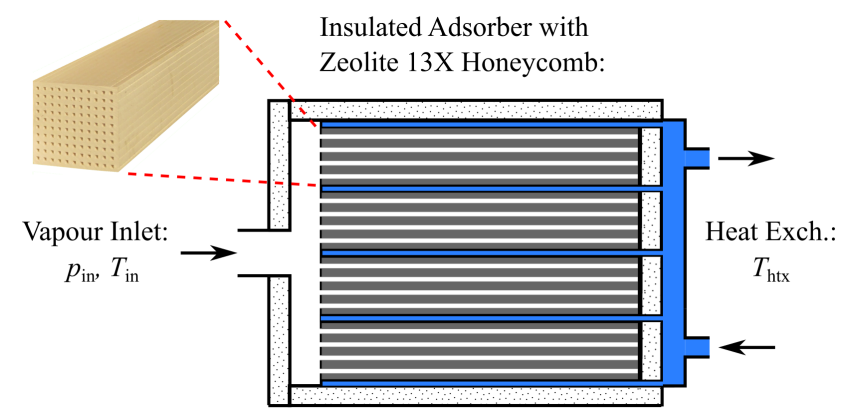

Figure 1: Examined adsorber set-up: stacked layers of honeycomb blocks, separated by heat exchanger plates to enhance the heat transfer between the adsorbent and the heat exchanger. (Photo of zeolite honeycomb block with courtesy of B. R. Formisano.) behaviour is discussed only qualitatively or by simple models. Further, our literature review found only very few publications on the detailed modelling and simulation of zeolite honeycomb adsorbers, e.g. [17]. In summary, all publications on honeycomb adsorbers solely examine open adsorption systems and assume a stationary flow of the carrier gas. In contrast, our work applies the detailed model presented in [7] to conduct simulations of the dynamic heat and mass transfer processes in a closed low-pressure honeycomb adsorber for thermal energy storage. In addition, we account for special effects of the rarefied gas flow, such as the slip-effect and thermal creep effect, which are neglected in most publications.

\subsection{Objectives of study}

The main objective of this study is to gain insight into the dynamic heat and mass transfer processes in a closed low-pressure honeycomb adsorber by means of numerical simulation. More specifically, the following questions regarding the modelling 
and the application are analysed:

Modelling:

- Is it valid to assume equilibrium in the adsorber for the temperature (isothermal), or pressure (isobaric), or the adsorption (local equilibrium of water uptake)?

- Is it necessary to take special effects of rarefied gas flow, such as the slip or thermal creep effect, into account?

Application:

- Is it possible to optimize the thermal power output of the adsorber by variation of the honeycomb geometry parameters (e.g. honeycomb width or channel size)?

- Is it possible to control the thermal power output?

In most publications the first question regarding the modelling is discussed only briefly. An extensive discussion of this question with respect to packed-bed adsorbers is given in [12] and [18]. The influence of geometry variation, here the particle size in a packed-bed, on the adsorption duration is studied in [12]. In summary, the questions specified above have not been discussed for the closed low-pressure honeycomb adsorber examined in this study.

\section{Model description}

\subsection{Modelling approach}

The examined adsorber set-up has been described briefly in Sect. 1.1 and is shown in Fig. 1. During the adsorption process, vapour flows into the adsorber by the vapour inlet. Given enough void space over the zeolite honeycomb arrangement, it can be assumed that the inflowing vapour distributes homogeneously over the zeolite honeycomb arrangement. This leads to a homogeneous pressure and temperature distribution over the whole honeycomb arrangement. Further, it is assumed, that the inlet pressure $p_{\text {in }}$ is constant and the temperature of the inflowing vapour is equal to the saturation temperature $T_{\text {in }}=T_{\mathrm{s}}\left(p_{\text {in }}\right)$, that is no limitations by the evaporator are considered. Furthermore, ideal thermal insulation of the adsorber is assumed.

Regarding the heat extraction, a more practical set-up is examined in this work in comparison to the basic set-up studied in [7]. The heat extraction is realized by heat exchanger plates between each layer of the stacked honeycomb blocks. In practical applications the temperature of the heat transfer fluid and thus of the heat exchanger surface would vary in time and along the adsorber. However, in this fundamental study, we apply a homogeneous and constant temperature $T_{\mathrm{htx}}$ over all heat exchanger plates. Hence, it is not required to model the heat and mass transfer in the heat exchanger plates.

The studied geometry of the identical honeycomb blocks is shown in Fig. 2. The aspect ratio of the outer height or width of a cut-out channel to the channel height or width is defined as

$\gamma_{a}:=\frac{a+2 f}{a}, \quad \gamma_{b}:=\frac{b+2 f}{b}$

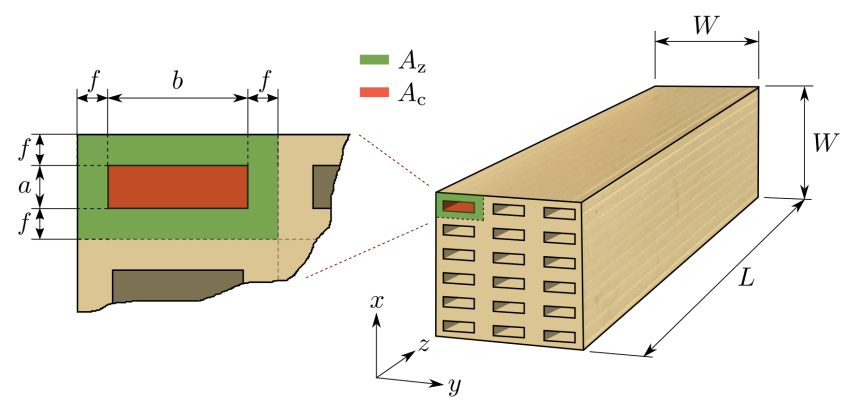

Figure 2: Geometry of the identical honeycomb blocks: honeycomb length $L$, honeycomb width $W$, channel height $a$, channel width $b$, (half) web thickness $f$, cross-section area of zeolite cut-out and channel $A_{\mathrm{z}}, A_{\mathrm{c}}$.

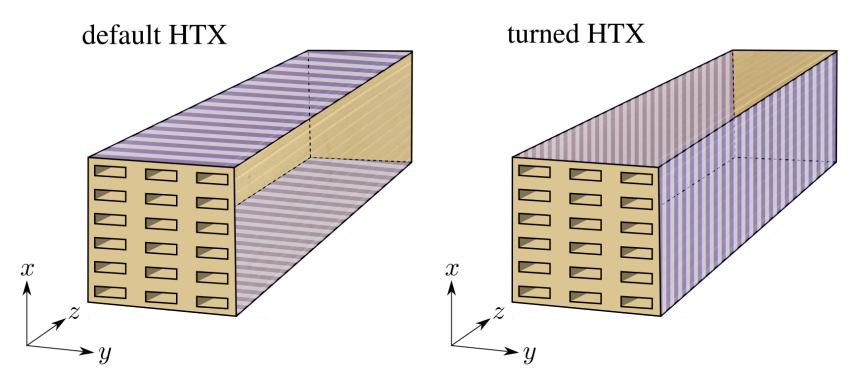

Figure 3: Two possible heat exchanger orientations: default and turned HTX. The heat exchanger boundaries are marked by striped shading.

For the general case of rectangular channels with $a \neq b$, the effective heat conductivity in the honeycomb cross-section is non-isotropic $\lambda_{\mathrm{eff}, x} \neq \lambda_{\mathrm{eff}, y}$. More precisely, for rectangular channels with $a \leq b$ the effective heat conductivity yields $\lambda_{\mathrm{eff}, x} \leq \lambda_{\mathrm{eff}, y}$. Hence, the orientation of the heat exchanger plates to the honeycomb blocks is of relevance for honeycomb blocks with non-square channels. The two possible heat exchanger orientations are given in Fig. 3.

From the description above and taking the assumptions into account it follows that the examined adsorber set-up leads to identical, two-dimensional fields of temperature and pressure in each honeycomb block. Thus, it is sufficient to analyse one column ( $z$ - $x$-plane) or one row ( $z-y$-plane) of channels depending on the orientation of the heat exchanger. Further, we assume that the solution has the same symmetry as the geometry and the boundary conditions of our problem and therefore restrict our consideration to one half of the honeycomb block. Regarding the heat transfer the boundary conditions are

$\left.\frac{\partial T}{\partial z}\right|_{z=0}=0,\left.\quad \frac{\partial T}{\partial z}\right|_{z=L}=0, \quad T(x=, y=W / 2)=T_{\mathrm{htx}}$.

With respect to the mass transfer the boundary conditions read

$p(z=0)=p_{\text {in }}, \quad \dot{m}(z=L)=0$,

where $\dot{m}$ denotes the mass flow of the vapour in the channels. Since the channels lead the vapour flow in $z$-direction, no boundary condition for the mass transfer is required at the heat exchanger surface for $x=W / 2$ or $y=W / 2$. The initial conditions are set to a homogeneous state of equilibrium

$T(t=0)=T_{0}, \quad p(t=0)=p_{0}, \quad X(t=0)=X_{\text {eq }}\left(p_{0}, T_{0}\right)$, 


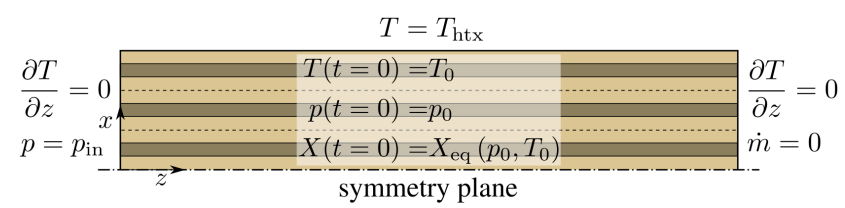

Figure 4: Reduced problem: Computation of the two-dimensional fields of temperature $T$, pressure $p$ and water uptake $X$ in one column of channels $(z-x-$ plane) with the given boundary and initial conditions.

with the water uptake being defined as

$X:=\frac{m_{\mathrm{a}}}{m_{\mathrm{z}}}$,

where $m_{\mathrm{a}}$ and $m_{\mathrm{z}}$ denote the mass of the adsorbate and the fully desorbed zeolite and $X_{\text {eq }}$ is the water uptake at equilibrium. In summary, given the boundary conditions by Eq. (2) and Eq. (3) and the initial conditions by Eq. (4) the three dependent variables temperature $T(\boldsymbol{x}, t)$, pressure $p(\boldsymbol{x}, t)$, and water uptake $X(\boldsymbol{x}, t)$ are computed, with $\boldsymbol{x}=(z, x)$ or $\boldsymbol{x}=(z, y)$ depending on the heat exchanger orientation. The reduced problem for the default orientation of the heat exchanger is depicted in Fig. 4 .

\subsection{Modelling equations}

The one-dimensional modelling equations of the heat and mass transfer and the adsorption for a single channel of a zeolite $13 \mathrm{X}$ honeycomb have been derived by the authors in [7]. The main assumptions of this one-dimensional model are:

- The vapour behaves as an ideal gas and as a viscous Newton-fluid with temperature dependent viscosity.

- The channel flow is assumed as creeping flow $(\operatorname{Re}<1)$.

- Rarefaction effects such as the slip-effect can be accounted for by implementing the so-called Poiseuille coefficient $G_{\mathrm{P}}$ into the Poiseuille equation.

- Locally ideal heat transfer between zeolite, adsorbate and vapour is assumed, resulting in one common temperature.

- The specific heat capacities, the effective heat conductivity and the porosity of the zeolite are assumed to depend on the temperature and the local water uptake.

- The heat conduction is modelled by the Fourier law.

- The adsorption kinetics can be described by the lineardriving-force approximation for a hollow cylindrical adsorbent.

- The adsorption equilibrium can be approximated by the Dubinin-Astakhov equation.

- The heat of adsorption depends on the water uptake.

For a more extensive discussion of the model and its assumptions we refer to [7].

Here, this one-dimensional model is applied to each channel in one column or row of the honeycomb block. To couple the channels thermally, a source/sink term $\sigma$ is added to the energy balance equation. Hence, the honeycomb is modelled as a continuum in longitudinal direction, whereas the model is discrete in transversal direction. Regarding the mass transfer, the equation for the mean vapour velocity in the channel $u$ is modified. With $\partial_{t}:=\partial \cdot / \partial t$ and $\partial_{z}:=\partial \cdot / \partial z$ the system of coupled partial differential equations can be written as

$$
\begin{gathered}
\left(\begin{array}{ccc}
\frac{1}{R_{\mathrm{s}} T} & -\frac{p}{R_{\mathrm{s}} T^{2}} & \frac{A_{\mathrm{z}}}{A_{\mathrm{c}}} \rho_{\mathrm{z}} \\
0 & \left(\rho_{\mathrm{z}} c_{\mathrm{z}}+\rho_{\mathrm{z}} X c_{\mathrm{a}}\right) & -\rho_{\mathrm{z}} \Delta h_{\mathrm{a}} \\
0 & 0 & 1
\end{array}\right)_{i} \cdot\left(\begin{array}{l}
\partial_{t} p \\
\partial_{t} T \\
\partial_{t} X
\end{array}\right)_{i}= \\
\left(\begin{array}{c}
\partial_{z}\left(\rho_{\mathrm{v}} \boldsymbol{u}\right) \\
\partial_{z}\left(\lambda_{\mathrm{eff}, z} \partial_{z} T\right)-\frac{A_{\mathrm{c}}}{A_{\mathrm{z}}} \rho_{\mathrm{v}} \boldsymbol{u} c_{p, \mathrm{v}} \partial_{z} T-\frac{A_{\mathrm{c}}}{A_{\mathrm{z}}} R_{\mathrm{s}} T \partial_{z}\left(\rho_{\mathrm{v}} \boldsymbol{u}\right)+\sigma \\
k_{\mathrm{a}}\left(X_{\mathrm{eq}}-X\right)
\end{array}\right)_{i},
\end{gathered}
$$

with

$i=1 \ldots N_{\mathrm{c}} / 2$,

where $i$ indicates the channel, starting from the channel at the heat exchanger boundary, and $N_{\mathrm{c}}$ denotes the numbers of channels in one column or row of one honeycomb block. The equations and values of the material functions and parameters can be found in [7]. The added and modified terms are marked bold in red and blue.

To calculate the mean vapour velocity in the channel $u$, the channel was transformed to a circular channel in [7] and the socalled Poiseuille coefficient $G_{\mathrm{P}}$ for a circular channel from [19] was implemented. Here, to increase the model accuracy especially for the rectangular channels with $a \neq b$, no transformation of the channels is conducted. Instead, the $G_{\mathrm{P}}$-function for rectangular channels is derived from the numerical data given in [20]. In addition, the thermal creep effect is taken into account. According to [20], the mean vapour velocity in a rectangular channel can be determined with the functions for the Poiseuille and thermal creep coefficients $G_{\mathrm{P}}$ and $G_{\mathrm{T}}$ by

$u=\frac{a}{\rho_{\mathrm{v}} \sqrt{2 R_{\mathrm{s}} T}}\left(-G_{\mathrm{P}}(\delta) \frac{\partial p}{\partial z}+G_{\mathrm{T}}(\delta) \frac{p}{T} \frac{\partial T}{\partial z}\right)$,

where $\delta$ denotes the rarefaction parameter. The rarefaction parameter is related to the Knudsen number $K n$ by

$\delta:=\frac{\sqrt{\pi}}{2} \frac{1}{K n} \quad$ with: $K n:=\frac{l_{\mathrm{mol}}}{a}$,

where $l_{\text {mol }}$ denotes the mean free path of the vapour molecules. The functions for $G_{\mathrm{P}}$ and $G_{\mathrm{T}}$ are determined by regression and given in Appendix A.1.

To couple the channels thermally, a source/sink term $\sigma$ is added to Eq. (6). The term accounts for the heat exchange between the channels due to heat conduction and is determined by

$\sigma_{i}=\frac{b+2 f}{A_{\mathrm{z}}} \cdot\left(\dot{q}_{i, i+1}-\dot{q}_{i, i-1}\right)$, 
where $\dot{q}_{i, i+1}$ and $\dot{q}_{i, i-1}$ are calculated as heat fluxes in analogy to Fourier's law. For $\dot{q}_{i, i+1}$ this approach yields

$\dot{q}_{i, i+1}=\frac{\lambda_{\mathrm{eff}, \mathrm{x}, i+1}+\lambda_{\mathrm{eff}, \mathrm{x}, i}}{2} \frac{T_{i+1}-T_{i}}{a+2 f}$

while for $\dot{q}_{i, i-1}$ the indices in Eq. (10) simply have to be reduced by one. To increase the model accuracy the heat flux at the heat exchanger boundary $\dot{q}_{\mathrm{htx}}=\dot{q}_{i, i-1}$ for $i=1$ is modelled by a one-sided approximation of 2 nd order which leads to

$\dot{q}_{\mathrm{htx}}=\lambda_{\mathrm{eff}, \mathrm{x}, 1} \frac{-T_{2}+9 T_{1}-8 T_{\mathrm{htx}}}{3(a+2 f)}$.

The effective heat conductivity $\lambda_{\mathrm{eff}, x}$ of each channel cut-out, is derived from a simple thermal resistance model and given in Appendix A.2. For the case of the turned heat exchanger, the channel width $b$ has to be substituted for the channel height $a$ and $x$ for $y$ and vice versa in Eq. (9), Eq. (10) and Eq. (11).

Finally, with respect to the model validation we refer to [7].

\section{Numerical implementation}

\subsection{Solution method}

As noted in the beginning of Sect. 2.2, the honeycomb is modelled as a continuum in longitudinal direction, whereas the model is discrete in transversal direction. To numerically solve the system of one-dimensional continuum equations (6) for each channel the finite-difference-method is implemented, applying the central difference approximation. Regarding the meshing in longitudinal direction, mesh studies yield that a knot spacing of $\Delta z=L / 250$ is sufficient, see Appendix B.

With respect to the transversal direction, the knot spacing is defined by the discrete model and is equal to the outer height or width of each channel $\Delta x=(a+2 f)$ or $\Delta y=(b+2 f)$. As a consequence, accurate results can only be achieved for cases with an adequate number of channels in a column or row, resulting in a transversal knot spacing $\Delta x$ or $\Delta y$ small enough in relation to the honeycomb width $W$. To evaluate whether the number of channels is adequate for achieving good numerical accuracy, a simulation can be conducted with higher order approaches for the heat flux terms in Eq. (9). If the results show no significant difference, the number of channels can be assumed adequate. The simulation of a reference case with the minimum number of channels $N_{\mathrm{c}}=4$ showed no significant variation of the results for 2nd- and 3rd-order approximations.

Regarding the time integration, the evaluation of the matrix on the left-hand side of the system of equations (6) reveals that the matrix elements range over several orders of magnitude $\left(10^{-9} \ldots 10^{9}\right)$, compare to [7]. Thus, the system can be expected to be stiff. The solver for stiff systems ode15s of Matlab (see [21] for solver description) is successfully applied. Finally, as the matrix is an upper triangular matrix the single equations can be sequentially evaluated in each time step.
Table 1: Varied geometry and process parameters of the studied simulation cases. The values of the reference case are underlined and marked in blue $\left(N_{\mathrm{c}}=16\right) .(\mathrm{BC}=$ boundary conditions; $\mathrm{IC}=$ initial conditions $)$

\begin{tabular}{|c|c|c|c|}
\hline & Parameter & Value & Unit \\
\hline \hline \multirow{4}{*}{} & $a$ & $0.5 ; \underline{\mathbf{1 . 0}} ; 2.0$ & $\mathrm{~mm}$ \\
\cline { 2 - 4 } & $b$ & $(\underline{\mathbf{1}} ; 2 ; 4 ; 10) \cdot a$ & $\mathrm{~mm}$ \\
\cline { 2 - 4 } & $f$ & $(0.25 ; 0.5 ; \mathbf{1}) \cdot a$ & $\mathrm{~mm}$ \\
\cline { 2 - 4 } & $L$ & $0.1 ; \underline{\mathbf{1 . 0}}$ & $\mathrm{m}$ \\
\hline \multirow{4}{*}{} & $W$ & $24 ; \underline{\mathbf{4 8}}$ & $\mathrm{mm}$ \\
\cline { 2 - 4 } & $T_{\mathrm{htx}}$ & $\underline{\mathbf{2 0}}$ & ${ }^{\circ} \mathrm{C}$ \\
\cline { 2 - 4 } & $p_{\mathrm{in}}$ & $\underline{\mathbf{1 0}} ; 20$ & $\mathrm{mbar}$ \\
\hline \multirow{4}{*}{$\circlearrowright$} & $T_{\mathrm{in}}=T_{\mathrm{s}}$ & $\underline{\mathbf{7}} ; 17.5$ & ${ }^{\circ} \mathrm{C}$ \\
\cline { 2 - 4 } & $T_{0}$ & $\underline{\mathbf{2 0}} ; 50$ & ${ }^{\circ} \mathrm{C}$ \\
\cline { 2 - 4 } & $p_{0}$ & $\underline{\mathbf{0 . 0 0 1}} ; 0.1$ & $\mathrm{mbar}$ \\
\cline { 2 - 4 } & $X_{0}=X_{\mathrm{eq}}$ & $0.055 ; \underline{\mathbf{0 . 1 0}} ; 0.15 ; 0.21$ & $\mathrm{~kg} / \mathrm{kg}$ \\
\hline
\end{tabular}

\subsection{Simulation case setup}

To study the influence of geometry variation, simulations are conducted for a relevant range of parameters. The typical channel size is in the order of $a=b=1 \mathrm{~mm}$ and the web thickness lies in the range of $f=0.1 \ldots 1 \mathrm{~mm}$, see [3]. Furthermore, the process is varied in terms of boundary and initial conditions, which are chosen in accordance to operating conditions for seasonal solar energy storage, e.g. [9]. The varied geometry and process parameters are summarized in Tab. 1. The values of a defined reference case are underlined and marked in blue. The implemented material parameters can be found in [7].

\section{Results and discussion}

\subsection{Reference case}

Before discussing the results regarding the specific questions formulated in Sect. 1.3, the reference case defined in Tab. 1 is studied to gain a basic understanding of the dynamic heat and mass transfer as well as adsorption processes in the examined closed low-pressure honeycomb adsorber. The evolution of the symmetric temperature $T(\boldsymbol{x}, \tilde{t})$, pressure $p(\boldsymbol{x}, \tilde{t})$ and water uptake $X(\boldsymbol{x}, \tilde{t})$ fields are shown for a half-section of the honeycomb block in Fig. 5. As noted in Sect. 2.2 and Sect. 3, the model and hence the results are discrete in transversal direction due to the discrete channels. However, in the figure the results are smoothed by interpolation in the transversal $x$-direction over the discrete channels. In addition, the thin horizontal lines mark the virtual boundaries between the discrete channels (compare the dashed lines in Fig. 4). Each row of the subfigures represents the state at a defined non-dimensional time

$\tilde{t}:=\frac{t}{t_{\mathrm{tot}}}$

where $t_{\text {tot }}$ denotes the total process duration. The total process duration is defined as the time required for the spatial variations of temperature, pressure and water uptake to decrease below $1 \%$ of the corresponding maximum values. 


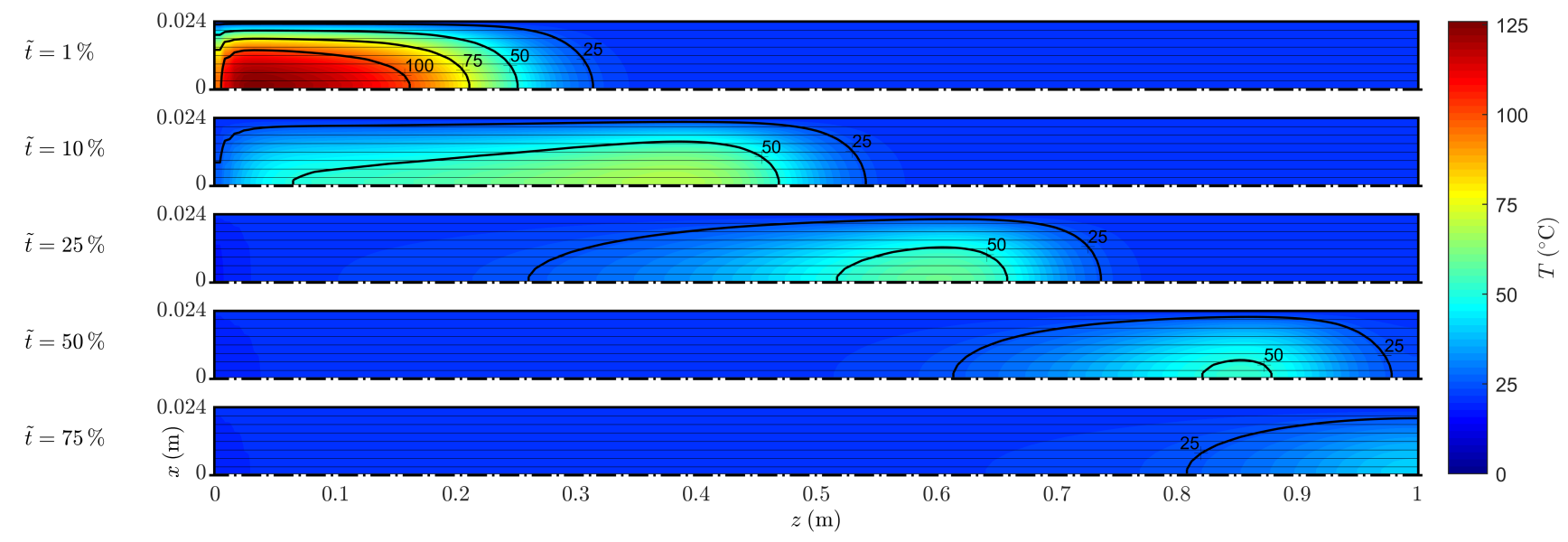

(a) Evolution of the symmetric temperature $T(\boldsymbol{x}, \tilde{t})$ field over the non-dimensional time $\tilde{t}$.

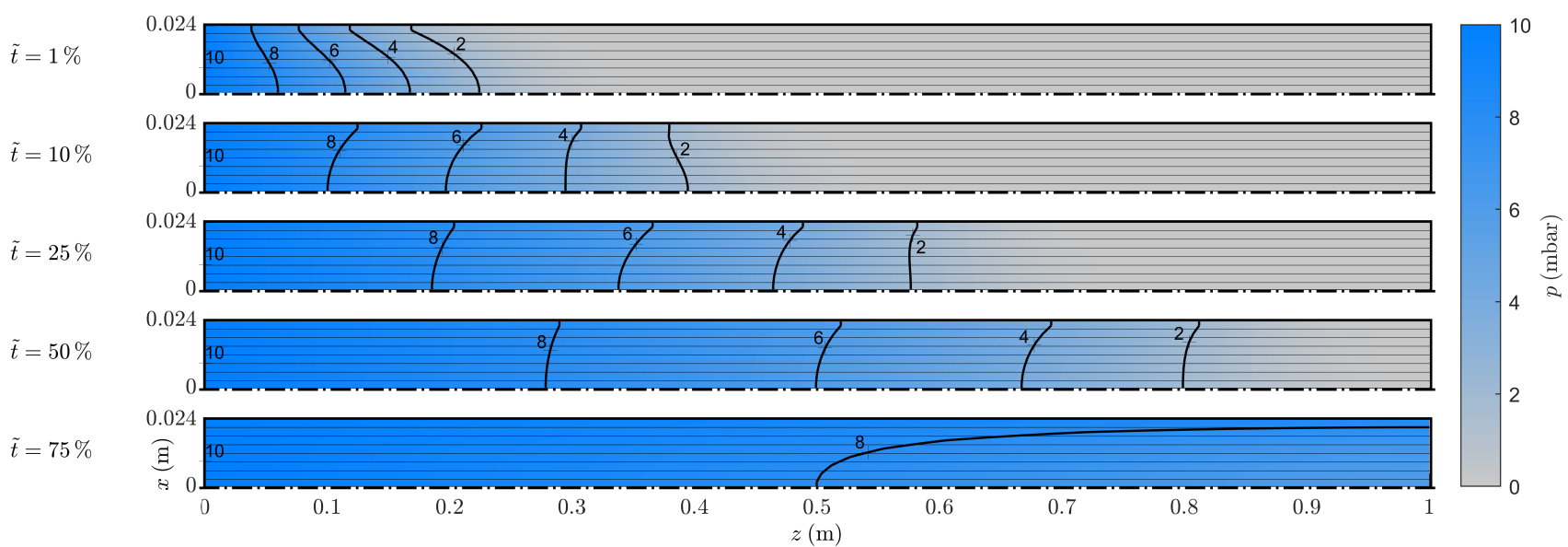

(b) Evolution of the symmetric pressure $p(\boldsymbol{x}, \tilde{t})$ field over the non-dimensional time $\tilde{t}$.

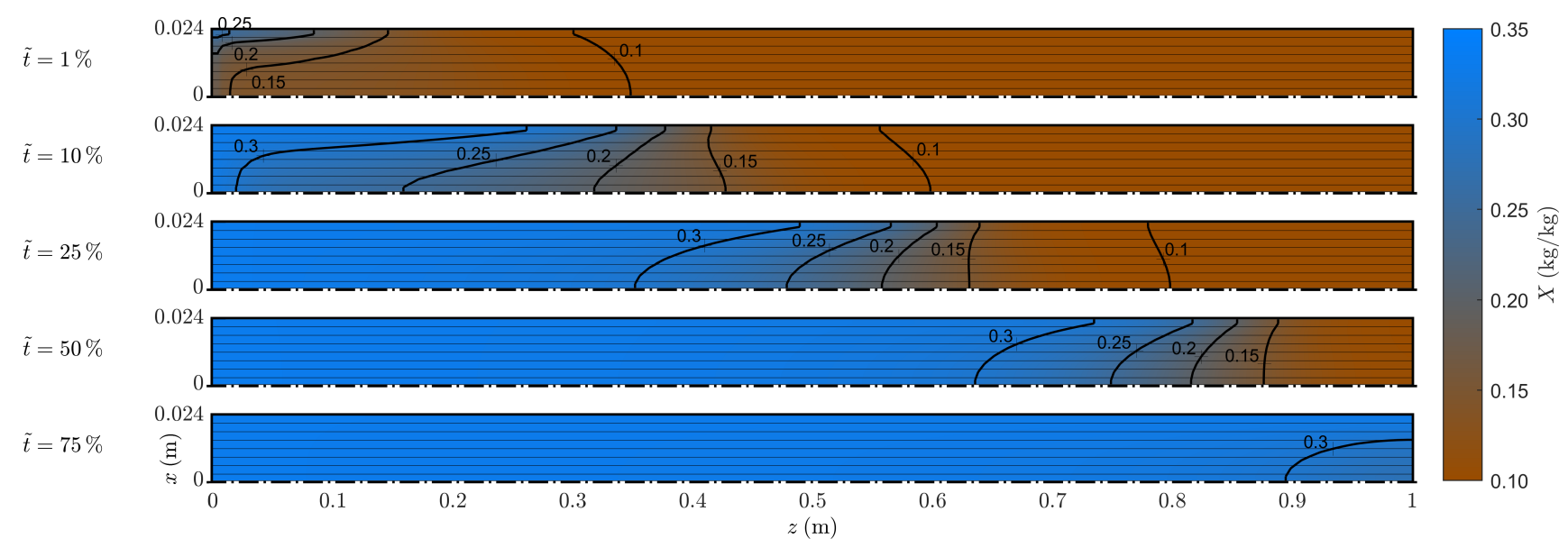

(c) Evolution of the symmetric water uptake $X(\boldsymbol{x}, \tilde{t})$ field over the non-dimensional time $\tilde{t}$.

Figure 5: Evolution of the symmetric temperature $T(\boldsymbol{x}, \tilde{t})$, pressure $p(\boldsymbol{x}, \tilde{t})$ and water uptake $X(\boldsymbol{x}, \tilde{t})$ fields over the non-dimensional time $\tilde{t}$ for a half-section of the honeycomb block corresponding to the reference case defined in Tab. 1 . The results are smoothened by interpolation in the transversal $x$-direction over the discrete channels. The thin horizontal lines mark the virtual boundaries between the discrete channels.

The analysis of the temperature reveals that the adsorption leads to a temperature wave moving through the honeycomb with the maximum values in the honeycomb centre and the minimum values at the heat exchanger boundary, see Fig. 5 (a). 
Initially, the central temperature rises to a considerably higher level of $T>100^{\circ} \mathrm{C}$ in comparison to the subsequent temperature evolution. Already after $10 \%$ of the total process duration the maximum temperature has decreased to $T<75^{\circ} \mathrm{C}$. Moreover, the advancing temperature wave has almost reached half of the honeycomb length. For the remaining time of the process the temperature peak gradually decreases while advancing further into the honeycomb before vanishing after it has reached the closed end of the honeycomb.

The observed temperature evolution can be understood from the evolution of the pressure field, see Fig. 5 (b). Initially, the applied boundary condition for the pressure at the inlet (Eq. (3), pressure jump) leads to a high pressure gradient, which induces a high vapour mass flow into the honeycomb and thus results in a high adsorption rate. In consequence, the temperature in the front part of the honeycomb rises quickly initially. Subsequently, as the inflowing vapour advances further into the honeycomb, the pressure gradient, and thus the vapour mass flow into the honeycomb and the adsorption rate, gradually decreases. Interestingly, at the beginning, the vapour advances fastest into the honeycomb at the centre, but after $25 \%$ of the total process duration the advancement of the vapour in the outmost channel at the heat exchanger boundary exceeds the central channel. As soon as the inflowing vapour reaches the closed end of the honeycomb, the pressure increases quickly to the inlet pressure throughout the honeycomb.

The last two effects described for the pressure evolution can be explained by the evolution of the water uptake field, see Fig. 5 (c). It is observed, that initially a significant rise of the water uptake $(X=0.15 \mathrm{~kg} / \mathrm{kg}$ ) occurs further into the honeycomb at the outer heat exchanger boundary compared to the honeycomb centre. This indicates that almost instantly the adsorption at the very front of the honeycomb centre reaches a temporary adsorption equilibrium. The temporary adsorption equilibrium results from the higher temperature reached in the honeycomb centre, due to the further distance to the heat exchanger boundary and thus lower cooling rate. As a result, a higher fraction of the inflowing vapour can bypass the front of the honeycomb centre without being adsorbed, thus resulting in the initially faster advancement of the vapour in the honeycomb centre. As soon as the temperature decreases again due to the heat extraction by the heat exchanger as well as convective cooling by the inflowing vapour, the adsorption continues in the front part of the honeycomb centre, and thus reduces the advancement of the vapour into the honeycomb.

The second effect of the fast rising pressure in the end of the process results from two events. First, as the vapour reaches the end of the honeycomb, the water uptake front also reaches the end. Thus, the mass sink due to adsorption vanishes, and hence, the inflowing vapour fills the channels faster. Secondly, as the vapour has reached the closed end of the honeycomb channels, a back-pressure effect occurs. Both events accelerate the pressure rise throughout the honeycomb at the end of the process.

The discussion of the reference case demonstrates that the heat and mass transfer and the adsorption processes are strongly coupled and can only be understood in their interaction. Finally, with respect to the studied cases with shorter honeycombs of
$L=0.1 \mathrm{~m}$, it is found, that the heat and mass transfer and adsorption processes are similar to the processes observed at the front $(z \leq 0.1 \mathrm{~m})$ of the reference case. Hence, no distinct temperature wave is observed for these cases.

\subsection{Modelling}

\subsubsection{Equilibrium assumption}

To discuss whether it would be valid to assume equilibrium in the adsorber for the temperature (isothermal) or the pressure (isobaric), the spatial variations have to be analysed. For this purpose, the maximum deviations from the maximum values in the adsorber can be evaluated. Regarding the adsorption, the maximum relative deviation from the corresponding local adsorption equilibrium has to be determined. The range of the maximum relative deviations of the temperature $T$, pressure $p$ and water uptake $X$ versus time in the studied adsorbers with different sets of geometry parameter but with common boundary and initial conditions corresponding to the reference case (see Tab.1) are shown in Fig. 6.

From Fig. 6(a) it follows that the relative deviations of the temperature do not exceed $30 \%$. On the other hand, the minimum deviations are around $5 \%$ for the first third of the process. Thus, in general, the spatial variations of the temperature should be accounted for. In addition, since in our model the thermal heat flux between the honeycombs and the heat exchanger is determined from the temperature gradient in the honeycomb at the heat exchanger surface, the spatial temperature distribution has to be considered in any case.

The evaluation of the relative deviations of the pressure yields that the range of the deviations can be distinguished by the honeycomb length, see Fig. 6(b). While for the honeycomb length of $L=1 \mathrm{~m}$ the spatial variations of the pressure should always be taken into account, the relative deviations of the pressure in a honeycomb with a length of $L=0.1 \mathrm{~m}$ decrease rapidly for few particular cases of large channel size $a$ and $b$ and low web thickness $f$. Hence, for this few particular cases the equilibrium assumption of an isobaric state in the adsorber could be applied. However, for most cases this oftenmade assumption should not be applied.

Regarding the relative deviations of the water uptake it is also found that the range of the deviations can be distinguished by the honeycomb length, see Fig. 6(c). Again, for a few particular cases the relative deviations of the water uptake in a honeycomb with a length of $L=0.1 \mathrm{~m}$ decrease rapidly. For most cases though, a significant deviation from the adsorption equilibrium over a relevant time is observed. Thus, local adsorption equilibrium should not be assumed for the studied cases.

In summary, the spatial variation of temperature and pressure as well as the local deviation from the adsorption equilibrium should be considered for the vast majority of the studied cases of the examined closed low-pressure honeycomb adsorber.

\subsubsection{Rarefaction effects}

As described in Sect. 2.2, our model takes rarefaction effects such as the slip effect and thermal creep effect into account. With respect to the thermal creep effect, the analysis 


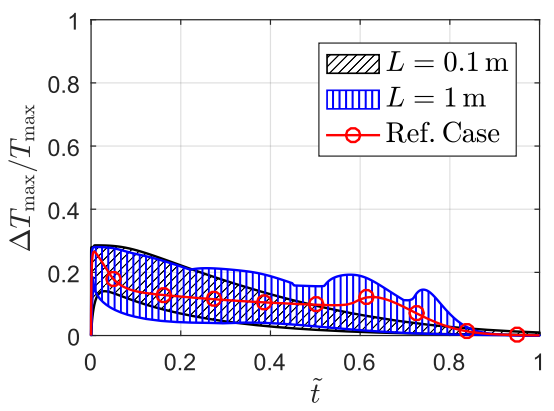

(a) Relative deviations of temperature $T$.

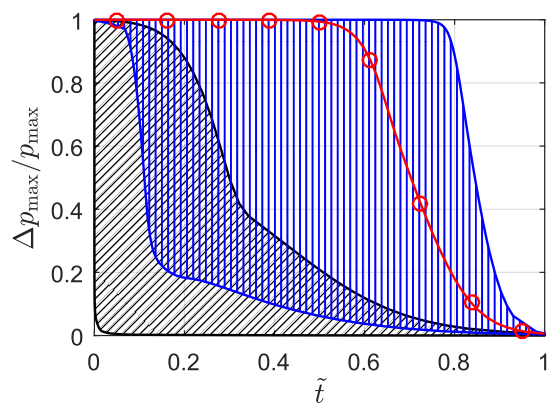

(b) Relative deviations of pressure $p$

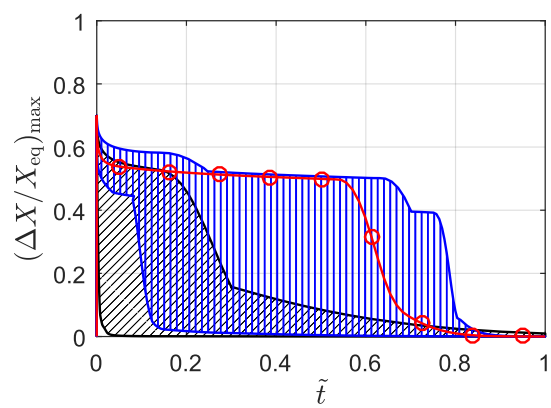

(c) Relative deviations of water uptake $X$.

Figure 6: Range of the maximum relative deviations of the temperature $T$, pressure $p$ and water uptake $X$ versus time in the studied adsorbers with different sets of geometry parameter but with common boundary and initial conditions corresponding to the reference case (see Tab.1).

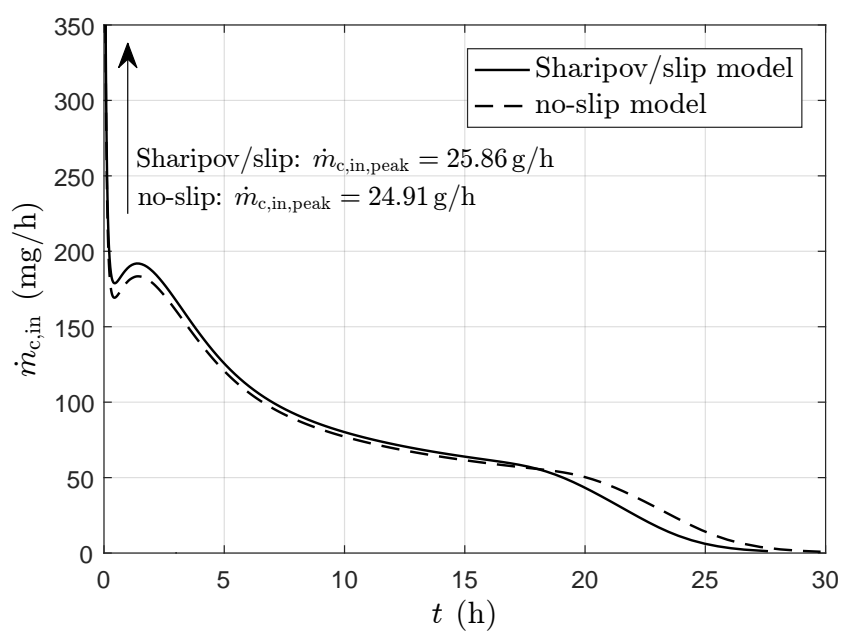

(a) Evolution of the vapour mass flow $\dot{m}_{\mathrm{c}, \text { in }}$ at the inlet of the central channel of the honeycomb. The peak value lies outside the range of the figure and is indicated by the vertical arrow.

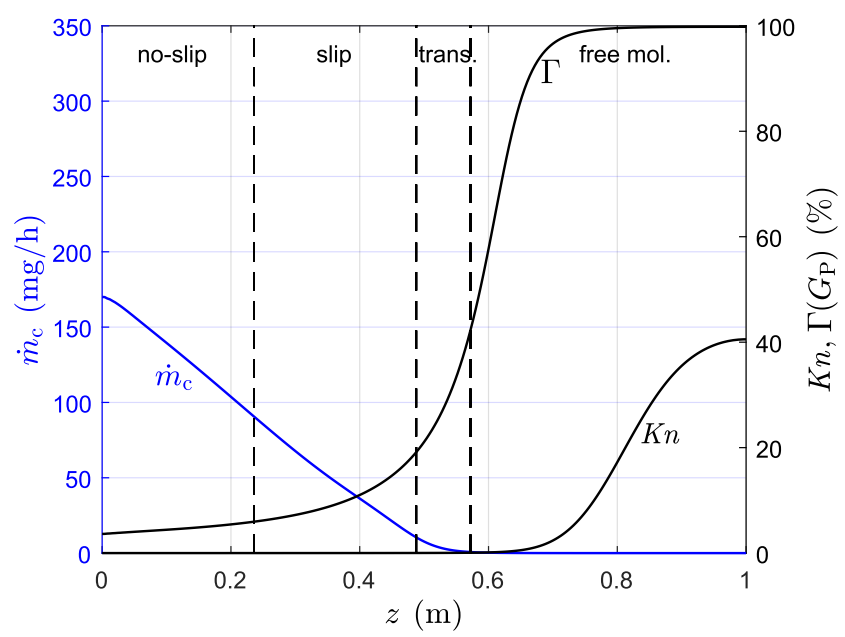

(b) Vapour mass flow $\dot{m}_{\mathrm{c}}$, Knudsen number $K n$ and relative error $\Gamma$ of the non-dimensional mass flow $G_{\mathrm{P}}$ for the no-slip model over the central channel of the honeycomb at $t=3 \mathrm{~h}$.

Figure 7: Influence of the rarefaction slip effect on the vapour mass flow in the central channel of the honeycomb for the reference case (see Tab. 1).

of the vapour mass flow yields that the mass flow induced by the thermal creep effect is smaller by approximately five orders of magnitude compared to the total mass flow in all studied cases. As an example, for the reference case, the maximum total mass flow into a single channel of the honeycomb is $25.86 \mathrm{~g} / \mathrm{h}$ comapred to a mass flow of $0.00033 \mathrm{~g} / \mathrm{h}$ induced by the thermal creep effect. In conclusion, the thermal creep effect is negligible for all studied cases, and thus the effect is not discussed further. However, the slip effect is found to have influence on the vapour mass flow in the honeycomb channels.

The evolution of the vapour mass flow $\dot{m}_{\mathrm{c}, \text { in }}$ at the inlet of the central channel of the honeycomb for the reference case is shown in Fig. 7 (a). Initially, as described for the reference case in Sect. 4.1, a high peak mass flow in relation to the subsequent course of the mass flow occurs. The peak value lies outside the range of the figure and is indicated by the vertical arrow. The no-slip model underestimates the peak mass flow by $3.8 \%$. Following, the mass flow rapidly decreases to a significantly lower level and after a small second peak continues to decrease gradually. The no-slip model continues to slightly underestimate the mass flow until $t \approx 17.5 \mathrm{~h}$. As a consequence, it predicts a higher mass flow afterwards, leading to an overestimation of the total process duration of about $8 \%$. The Sharipov and the slip model, corresponding to the $G_{\mathrm{P}}$-functions (A.1) and (A.2), yield identical results over the whole process duration. Thus, rarefaction effects beyond the slip effect seem negligible. Finally, since the total thermal power of the adsorber is proportional to the vapour mass flow into the adsorber, it can be concluded that the no-slip model also predicts the evolution of total thermal power fairly well.

To evaluate the local influence of the slip effect, the vapour mass flow $\dot{m}_{\mathrm{c}}$ (blue) over the central channel of the honeycomb for the reference case is examined for $t=3 \mathrm{~h}$, see Fig. 7 (b). The linear decrease of the mass flow indicates that the inflowing vapour is adsorbed with a constant adsorption rate over half of the honeycomb length for the particular moment. Hence, adsorption not only occurs at a sharp adsorption front. Besides the mass flow, also the Knudsen number $K n$ with the corresponding flow regimes as well as the relative error $\Gamma$ of the nondimensional mass flow $G_{\mathrm{P}}$ determined by the no-slip model is 
depicted. The figure reveals that the relevant mass flow reaches from the no-slip over the slip into the transitional flow regime, leading to an increasing local error of the non-dimensional mass flow of more than $40 \%$ at the adsorption front. However, this high local error of the non-dimensional mass flow is compensated to some extent by another error of the no-slip model regarding the pressure curve. In general, the no-slip model yields a more convex pressure curve over the channel, resulting in higher pressure gradients further into the channel. Thus, according to Eq. (7), the underestimation of the non-dimensional mass flow is partially compensated by the overestimation of the pressure gradient. It is found, that the accumulated local error of the temperature at the examined time reaches up to $10 \%$ and smears the sharp temperature front.

\subsection{Application}

For TES application, the thermal power is of great interest. Therefore, the following discussions related to the application focus on the thermal power. For better comparison of the different cases, the volumetric thermal power is examined.

\subsubsection{Geometry variation}

At first, the effect of geometry variation on the thermal power is studied for the special case of honeycombs with square channels, $a=b$. In this case, $\gamma=\gamma_{a}=\gamma_{b}$ with $\gamma_{a}$ and $\gamma_{b}$ according to Eq. (1) applies. Relating $\gamma$ to the porosity $\varepsilon$ of the honeycomb yields

$\varepsilon=\frac{1}{\gamma_{a} \gamma_{b}}=\frac{1}{\gamma^{2}}$

The dependency of the volumetric thermal power $p_{\text {th }}$ on the honeycomb geometry for the special case of honeycombs with square channels and the boundary and initial conditions of the reference case is depicted in Fig. 8. While the absolute thermal powers for the different honeycomb lengths lie in the same range, the volumetric power of the honeycomb with $L=1 \mathrm{~m}$ is approximately a tenth of the volumetric power of the honeycomb with $L=0.1 \mathrm{~m}$. As expected, the reduction of the honeycomb width $W$ increases the thermal power, due to the reduction of the heat transfer resistance. In addition, it is found, that the increase of $\gamma$, that is the reduction of the honeycomb porosity $\varepsilon$, leads to a reduction of the thermal power, caused by an increase of the internal mass transfer resistance of the micro-porous zeolite due to an increased web thickness $f$.

In Fig. 8 (a), the peak power $p_{\text {th,peak }}$ is shown. Similar to [12] which found a local minimum of the cycle duration over the adsorbent particle size in a closed adsorption heat pump, we find an optimum of the peak power over the channel size for a honeycomb length of $L=0.1 \mathrm{~m}$. The local optimum can be explained by the external and internal mass transfer resistance. Left of the optimum the thermal power is limited by the external mass transfer resistance of the honeycomb channels. Increasing the channel size reduces the external mass transfer resistance and thus increases the thermal power. At the same time, for a fixed $\gamma$, that is a fixed porosity $\varepsilon$ of the honeycomb, the increase of the channel size also leads to an increase of the honeycomb web thickness $f$. As a consequence, while the external mass transfer resistance reduces with increasing channel size, the internal mass transfer resistance of the micro-porous zeolite increases. Hence, right of the optimum the thermal power is limited by the internal mass transfer resistance of the micro-porous zeolite. For a honeycomb length of $L=1 \mathrm{~m}$, no local optimum of the peak power exists in the depicted range of channel size. The optimum is found to be around $a \approx 4 \mathrm{~mm}$.

The evaluation of the mean power yields the interesting result that, while an optimum of the peak power is found for a honeycomb length of $L=0.1 \mathrm{~m}$, no optimum exists for the mean power in the examined range of the channel size, see Fig. 8 (b). For the honeycomb length of $L=1 \mathrm{~m}$ the opposite is found. The explanation of the optimum of the mean power is analogue to the explanation of the peak power optimum. In summary, the main conclusion is that TESs based on the examined closed low-pressure honeycomb adsorber can be optimized either for the peak or the mean power.

To complete the discussion on the geometry variation, the thermal power is examined for the general case of honeycombs with non-square channels. The volumetric thermal power $p_{\text {th }}$ versus time for the reference case and for different cases of honeycombs with $a \neq b$ is shown in Fig. 9. It is found that the peak power increases with decreasing aspect ratio $a / b$, given the constant value of $a=1 \mathrm{~mm}$ of the channel height. The increase of the peak power results from the decrease of the external mass transfer resistance of the honeycomb channels with increasing channel width $b$. With respect to the heat exchanger orientation no significant difference between the configurations occurs for $a / b=1 / 2$, see Fig. 3 . For the aspect ratio of $a / b=1 / 10$ the configuration with the turned heat exchanger leads to an increase of the peak power by $\approx 20 \%$. Finally, no further increase of the thermal power with the channel width can be expected, since the external mass transfer resistance for $a / b=1 / 10$ is already close to the mass transfer resistance of the planar Poiseuille flow.

\subsubsection{Power control}

From the analysis of the reference case in Sect. 4.1 it followed that the boundary condition of the pressure at the inlet (Eq. (3), pressure jump) initially induces a high vapour inflow resulting in the high temperature rise at the honeycomb front. This temperature rise leads to an initial peak of the thermal power, compare e. g. Fig. 9. In conclusion, to control the thermal power, the inlet pressure has to be controlled.

The implementation of a standard proportional-integralderivative controller (PID-controller, see [22]) suggests itself. Unfortunately, restrictions by the applied time-integration solver (odes15s from Matlab, see Sect. 3.1) do not allow for the application of a PID-controller. However, the implementation of an integral controller (I-controller) is straightforward. To overcome the usual limitation of the dynamics of an Icontroller, a simple adaptive I-controller is designed. The controller equation reads

$$
\frac{\partial p_{\text {in }}}{\partial t}=\min \left(K_{\mathrm{I}, 1}\left(p_{\text {in }, \max }-p_{\text {in }}(t)\right) ; K_{\mathrm{I}, 2}\left(p_{\text {th, set }}-p_{\text {th }}(t)\right)\right) .
$$




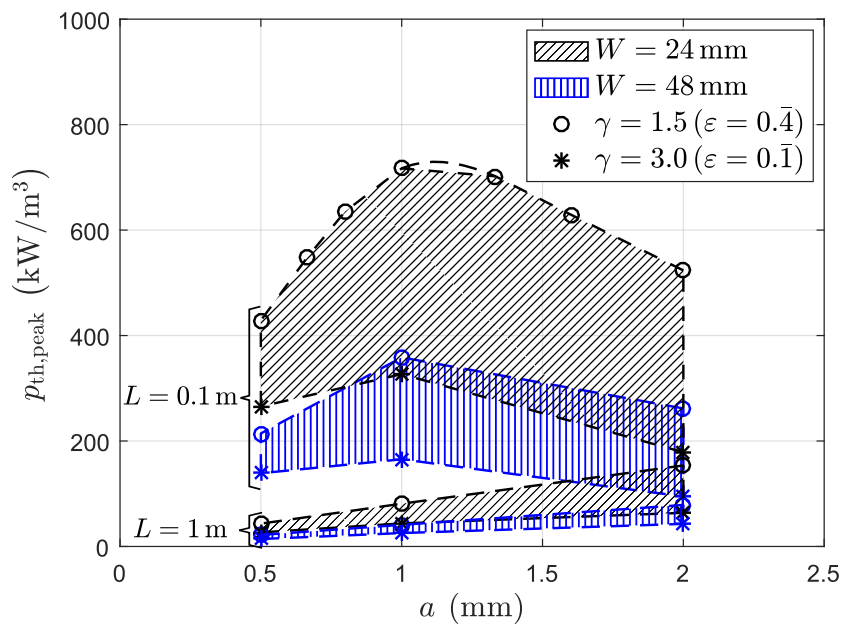

(a) Peak thermal power.

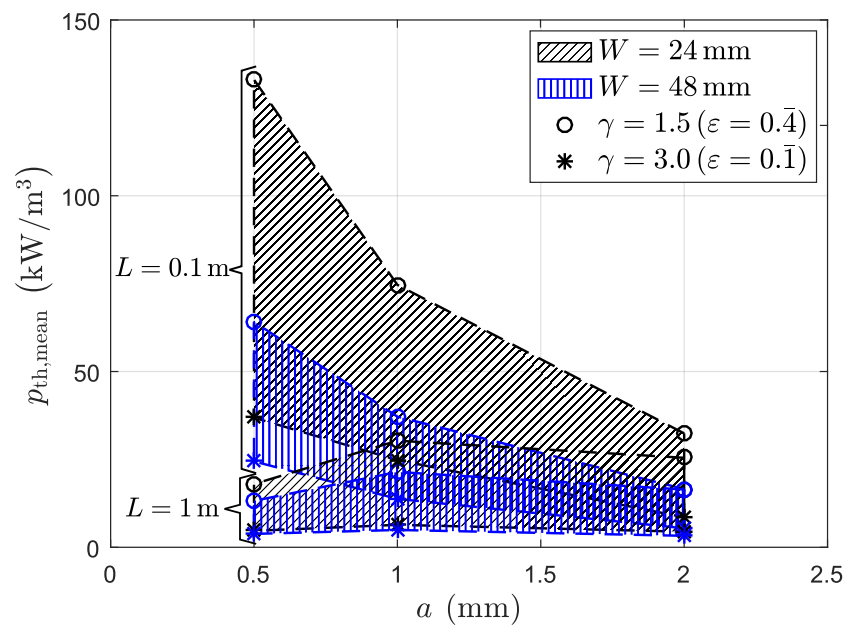

(b) Mean thermal power.

Figure 8: Dependency of the volumetric thermal power $p_{\text {th }}$ on the honeycomb geometry for the special case of honeycombs with square channels and the boundary and initial conditions of the reference case (see Tab. 1). The optima of the volumetric thermal power result from the external and internal mass transfer resistances.

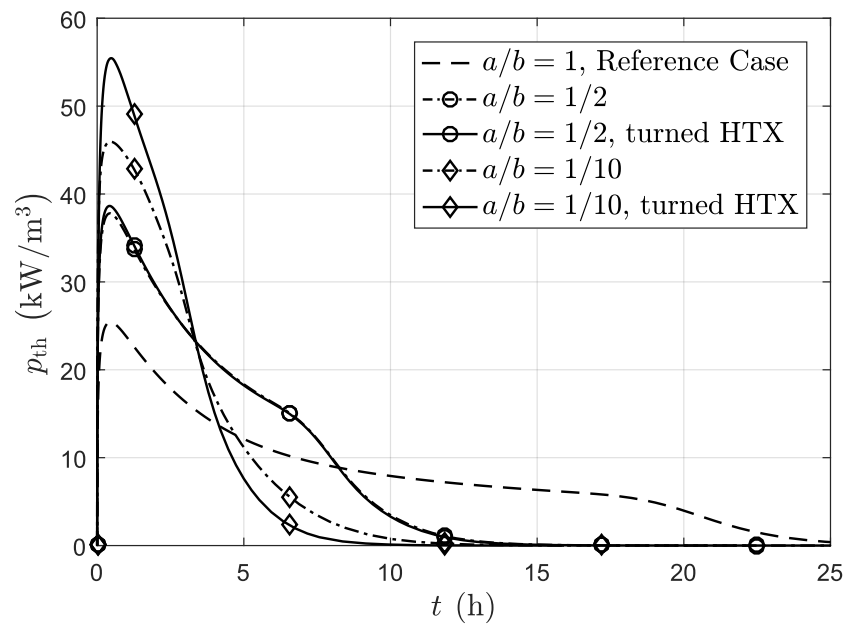

Figure 9: Volumetric thermal power $p_{\text {th }}$ versus time for the reference case (see Tab. 1) and different cases of honeycombs with $a \neq b$.

The controller contains two controller equations, where the first equation defines a pressure regulated controller and the second equation defines a power regulated controller. Initially the pressure regulated controller is effective, leading to a fast increase of the inlet pressure. As soon as the thermal power is close to the set point $p_{\text {th,set }}$ the power regulated controller applies, strongly reducing the increase rate of the pressure, and thus avoiding a significant overshoot of the controlled power. The controller is found to be robust and only of low sensitivity to variation of the controller parameters. As suitable controller parameters the values $K_{\mathrm{I}, 1}=10^{-4}$ and $K_{\mathrm{I}, 2}=10^{-1}$ are identified.

The evolution of the volumetric thermal power for the controlled cases of different set point values $p_{\text {th,set }}$ and the notcontrolled reference case is shown in Fig. 10. The results show that the thermal power can be effectively controlled by shifting the thermal energy of the power peak. For the third con-

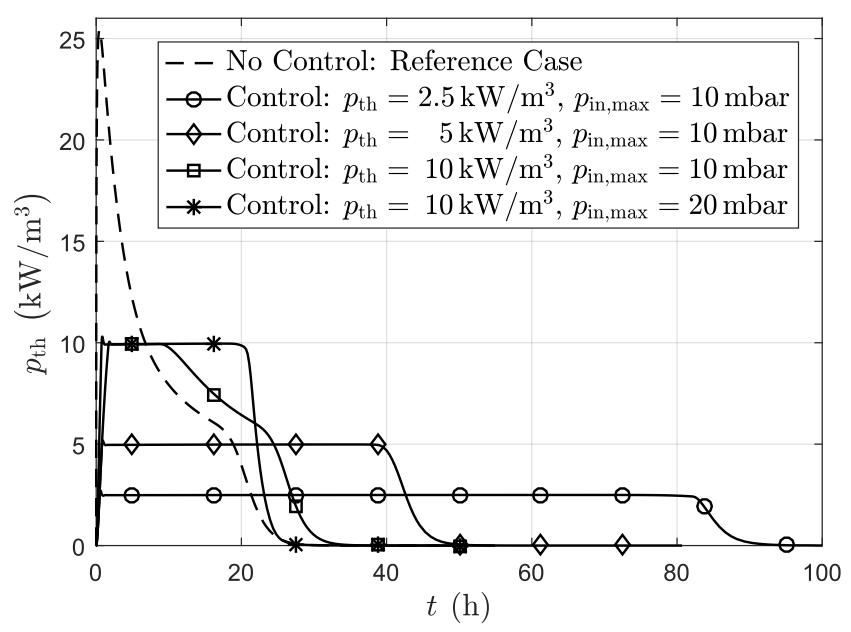

Figure 10: Volumetric thermal power $p_{\text {th }}$ versus time for controlled cases of different set point values $p_{\text {th,set }}$ and the not-controlled reference case (see Tab. 1).

trolled case with a set point value $p_{\text {th,set }}=10 \mathrm{~kW} / \mathrm{m}^{3}$ and a maximum inlet pressure of $p_{\text {in,max }}=10 \mathrm{mbar}$, it is found that the thermal power can not be hold at the set point value for the total process duration. For $t>10 \mathrm{~h}$ the thermal power becomes limited by the mass transfer. One solution to overcome the limitation by the mass transfer is to increase the inlet pressure. The forth case with a maximum inlet pressure of $p_{\text {in,max }}=20$ mbar proves that the power can be hold at the set point value of $p_{\text {th,set }}=10 \mathrm{~kW} / \mathrm{m}^{3}$ for the total process duration.

The evolution of the controlled inlet pressure $p_{\text {in }}$ for the controlled cases of different set point values $p_{\text {th,set }}$ and the notcontrolled reference case is shown in Fig. 11. The results show that the inlet pressure initially increases quickly to a value proportional to the set point value $p_{\text {th,set }}$. As soon as the set point value is reached the power regulated controller is applied, sig- 


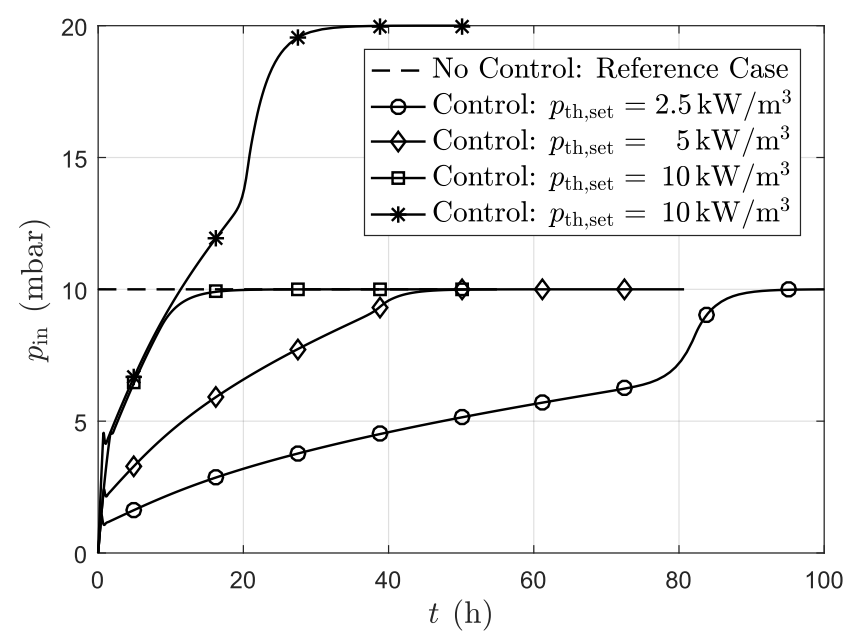

Figure 11: Controlled inlet pressure $p_{\text {in }}$ versus time for controlled cases of different set point values $p_{\text {th,set }}$ and the not-controlled reference case (see Tab. 1).

nificantly reducing the increase rate of the pressure. Again, the slope of the subsequent pressure curve is proportional to the set point value of the thermal power. The increasing slope at the end of the pressure curve for the first and last controlled cases indicates, that the pressure regulated controller becomes activated again since the power can no longer be hold at the set point value.

Finally, the evolution of the symmetric temperature $T(\boldsymbol{x}, \tilde{t})$ field for the controlled case with a set point value of $p_{\text {th,set }}=$ $10 \mathrm{~kW} / \mathrm{m}^{3}$ and a maximum inlet pressure of $p_{\mathrm{in}, \max }=20 \mathrm{mbar}$ is evaluated for a half-section of the honeycomb block in Fig. 12. The comparison with the evolution of the symmetric temperature $T(\boldsymbol{x}, \tilde{t})$ field for the not-controlled reference case (see Fig. 5) confirms the assumption that the controlled increase of the inlet pressure prevents the initial overheating of the honeycomb front. Furthermore, as the temperature wave advances into the honeycomb, the temperature peak remains at the same level resulting in a constant thermal power. Finally, the second and last row show a longer temperature zone with $T \geq 50^{\circ} \mathrm{C}$. This longer zone compensates that initially and at the end of the process the total length of the zone of increased temperature in the honeycomb is slightly shorter compared to the wave length of the advancing wave.

\section{Conclusion and outlook}

A closed low-pressure honeycomb adsorber with zeolite 13X for thermal energy storage has been examined numerically. A practical adsorber set-up has been studied, applying the onedimensional model of a single channel of the honeycomb presented in [7]. The introductory discussion of a reference case demonstrated that the heat and mass transfer and the adsorption processes are strongly coupled and can be only understood in their interaction. With respect to the specific questions formulated in Sect. 1.3 following results can be summarized: Modelling:
- The spatial variations of temperature and pressure as well as the local deviation from adsorption equilibrium are found to be significant for the vast majority of studied cases. Only for a few cases with a honeycomb length of $L=0.1 \mathrm{~m}$ and large channel size $a$ and $b$ and low web thickness $f$, it is legit to assume an isobaric state and local adsorption equilibrium.

- The rarefaction effect in terms of the slip effect has only minor influence on the evolution of the vapour mass flow and leads to a reduction of the total process duration of about $8 \%$ for the reference case. The thermal creep effect effect is found to be negligible for all studied cases.

\section{Application:}

- The analysis of the volumetric thermal power yields that the power can be increased in general, by reducing the honeycomb length and width as well as the web thickness, that is increasing the honeycomb porosity. For a honeycomb length of $L=0.1 \mathrm{~m}$ and a fixed porosity, the peak power can be optimized by variation of the channel size in the studied range. The optimum is a result of the external and internal mass transfer resistance decreasing and increasing respectively with the channel size. With respect to the mean power, an optimum is found for a honeycomb length of $L=1 \mathrm{~m}$. Interestingly, the optimum for peak and mean power do not coincide in general.

- The thermal power can be effectively controlled by the inlet pressure. The regulation of the inlet pressure prevents the initial overheating of the honeycomb front and shifts this thermal energy in time. The reference case yields that for high set point values of the power the process becomes limited by the mass transfer. This limitation can be overcome by increasing the maximum inlet pressure.

With respect to further studies, a homogenized macro-scale model should be derived from the present model to study packed-bed adsorbers with small particles (e.g. zeolite powders) for TES application. It can be expected that the rarefaction effects are significant for this type of adsorbers. On the other end of the scale, three-dimensional pore-scale simulations of the external and internal heat and mass transfer process should be conducted to proof the physical accuracy of the macro-scale model. Finally, it should be emphasized, that our model is not limited to zeolite $13 \mathrm{X}$ adsorbents and could be applied to adsorbers with other adsorbent materials.

\section{Acknowledgment}

The authors are grateful to M. Farahani and J. Vogel for fruitful discussions on numerical issues. 


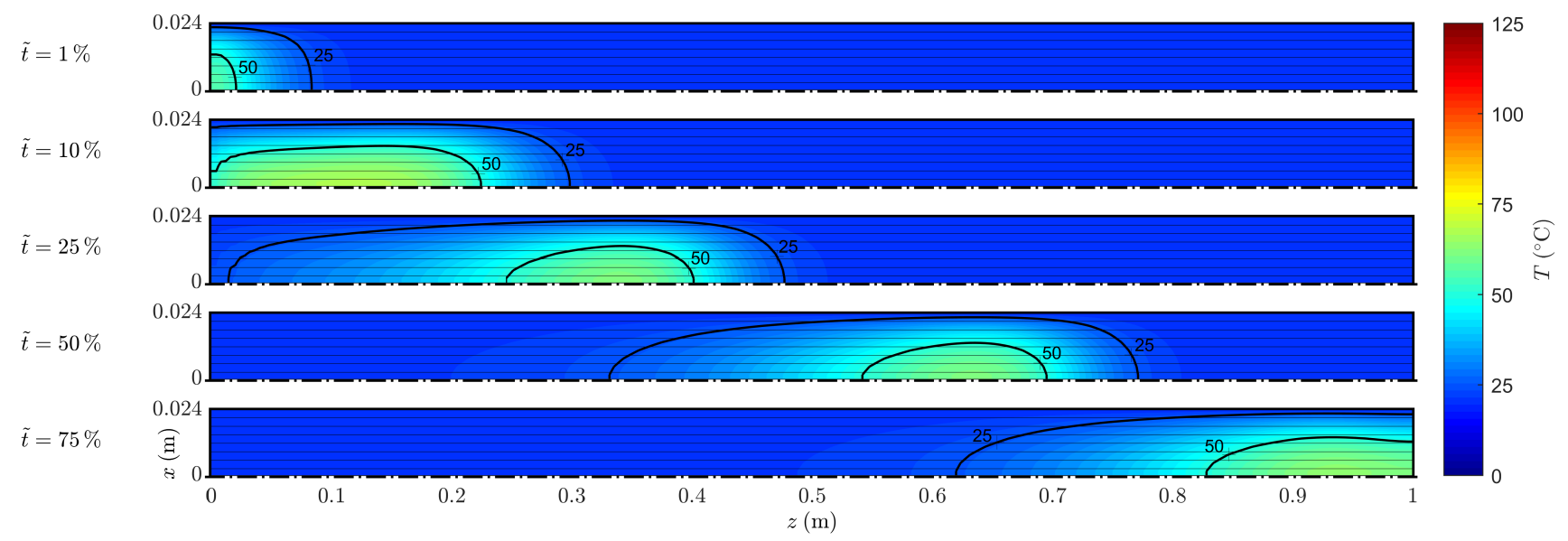

Figure 12: Evolution of the symmetric temperature $T(\boldsymbol{x}, \tilde{t})$ field for the controlled case with a set point value of $p_{\text {th,set }}=10 \mathrm{~kW} / \mathrm{m}^{3}$ and a maximum inlet pressure of $p_{\text {in,max }}=20$ mbar for a half-section of the honeycomb block. The results are smoothened by interpolation in the transversal $x$-direction over the discrete channels. The thin horizontal lines mark the virtual boundaries between the discrete channels.

\section{Appendix A. Model Parameters}

Appendix A.1. Poiseuille and thermal creep coefficient

For the special case of a rectangular channel with $a=b$, we determine the following $G_{\mathrm{P}}$-function via regression

$$
\begin{aligned}
G_{\mathrm{P}}=\frac{0.8372-0.1021 \delta^{0.8747} \ln (\delta)}{1+3.032 \delta^{0.9376}} & \\
& +\left(\frac{\delta}{14.227}+0.7036\right) \frac{\delta}{0.3652+\delta} .
\end{aligned}
$$

For the general case of a rectangular channel with $a \neq b$ similar functions were determined. With respect to the results discussed in Sect. 4.2.2, it is valid to use the simpler slip approach. This leads to the substantially shorter form of the $G_{\mathrm{P}}$-function

$$
G_{\mathrm{P}}=\zeta_{1} \delta+\zeta_{2}
$$

The values of the parameters $\zeta_{i}$ for different aspect ratios $a / b$ of a rectangular channel are summarized in Tab. A.2.

Table A.2: Parameters $\zeta_{i}$ of the $G_{\mathrm{P}}$-function (A.2) with the slip approach of 1st order for different aspect ratios $a / b$ of a rectangular channel.

\begin{tabular}{|c|c|c|}
\hline$a / b$ & $\zeta_{1}$ & $\zeta_{2}$ \\
\hline \hline 1 & 0.0703 & 0.5713 \\
\hline $1 / 2$ & 0.1143 & 0.7607 \\
\hline $1 / 4$ & 0.1404 & 0.8872 \\
\hline $1 / 10$ & 0.1562 & 0.9645 \\
\hline
\end{tabular}

Regarding the thermal creep coefficient $G_{\mathrm{T}}$, the following function was found to fit the numerical data from [20] well

$G_{\mathrm{T}}=\left(\xi_{1} \delta+\frac{\xi_{2}+\xi_{3} \delta}{\xi_{4}+\xi_{5} \delta}\right)^{-1}$

with $\xi_{i}$ as fitting parameters. The values of the parameters $\xi_{i}$ for different aspect ratios $a / b$ of a rectangular channel are summarized in Tab. A.3.
Table A.3: Parameters $\xi_{i}$ of the $G_{\mathrm{T}}$-function (A.3) for different aspect ratios $a / b$ of a rectangular channel.

\begin{tabular}{|c|c|c|c|c|c|}
\hline$a / b$ & $\xi_{1}$ & $\xi_{2}$ & $\xi_{3}$ & $\xi_{4}$ & $\xi_{5}$ \\
\hline \hline 1 & 0.837 & 4.989 & 31.92 & 2.088 & 9.904 \\
\hline $1 / 2$ & 0.8473 & 6.2 & 48.32 & 3.541 & 19.79 \\
\hline $1 / 4$ & 0.8567 & 2.09 & 17.03 & 1.546 & 7.864 \\
\hline $1 / 10$ & 0.872 & 3.784 & 44.32 & 3.711 & 21.59 \\
\hline
\end{tabular}

\section{Appendix A.2. Effective heat conductivity}

The effective heat conductivity $\lambda_{\mathrm{eff}, x}$ or $\lambda_{\mathrm{eff}, y}$ of a single channel cut-out, is determined from a simple thermal resistance model, see Fig. A.13.

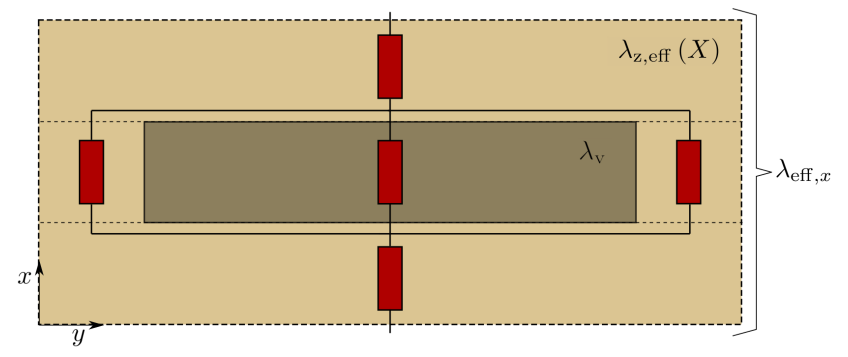

Figure A.13: Thermal resistance model to determine the effective heat conductivity $\lambda_{\mathrm{eff}, x}$ of a single channel cut-out. For the geometry parameters see Fig. 2.

Analysis of the thermal resistance model yields

$$
\lambda_{\mathrm{eff}, x}=\lambda_{\mathrm{z}, \mathrm{eff}}(X) \frac{\gamma_{a}}{\left(\gamma_{a}-1\right)+\gamma_{b}\left(\left(\gamma_{b}-1\right)+\lambda_{\mathrm{v}} / \lambda_{\mathrm{z}, \mathrm{eff}}(X)\right)^{-1}},
$$

with $\gamma_{a}$ and $\gamma_{b}$ as defined in Eq. (1) and the effective heat conductivity of the zeolite

$$
\lambda_{\mathrm{z}, \mathrm{eff}}(X)=\left(1-\varepsilon_{\max }\right) \lambda_{\mathrm{z}}+\frac{\rho_{\mathrm{z}}}{\rho_{\mathrm{a}}} X \lambda_{\mathrm{a}}+\varepsilon(X) \lambda_{\mathrm{v}} .
$$


Here, $\varepsilon$ denotes the micro-porosity of the zeolite. The equations and values of the material functions and parameters can be found in [7]. For the case of the turned heat exchanger, the channel width $b$ has to be substituted for the channel height $a$ and vice versa in Eq. (A.4) to determine $\lambda_{\mathrm{eff}, y}$.

\section{Appendix B. Numerical implementation}

To examine the longitudinal mesh convergence, three indicator variables are defined. The convergence of the temperature is analysed by the maximum temperature $T_{\max }$. For the pressure the duration of the inflowing vapour to reach the end of the central channel $t_{p, 10}=t\left(p(L)=p_{\text {in }} / 10\right)$ is examined. The convergence of the water uptake is analysed by the maximum deviation from the equilibrium water uptake $\Delta X_{\max }$. The results of four different test cases, which vary significantly in their mass transfer dynamics, yield that a knot spacing of $\Delta z_{\min }=L / 250$ is sufficient. As an example, the convergence plot of the maximum temperature $T_{\max }$ for one test case is given in Fig. B.14.

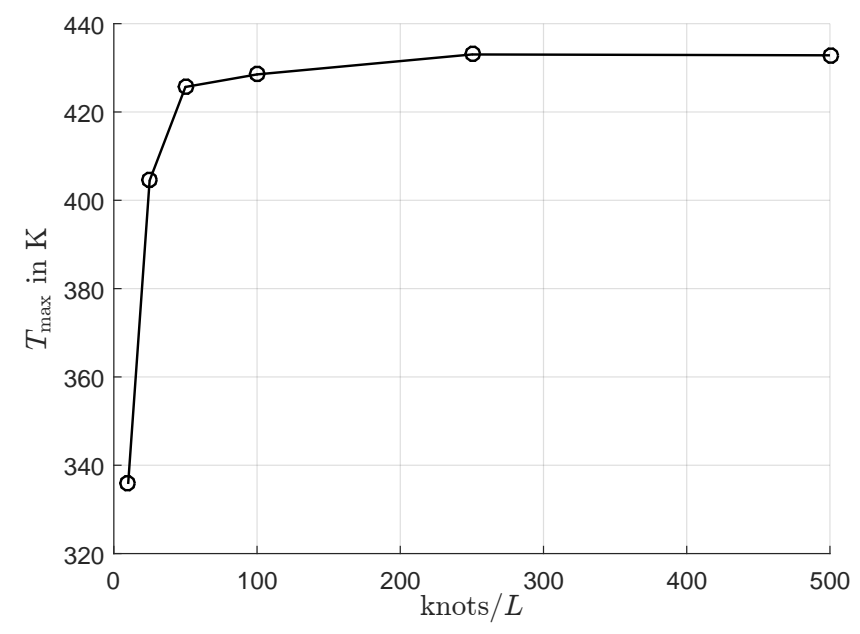

Figure B.14: Mesh convergence of the maximum temperature $T_{\max }$ for the test case: $a=b=0.5 \mathrm{~mm}, f=0.5 \mathrm{~mm}, L=1 \mathrm{~m}, W=24 \mathrm{~mm}\left(N_{\mathrm{c}}=16\right), T_{\mathrm{htx}}=$ $20^{\circ} \mathrm{C}, p_{\text {in }}=10 \mathrm{mbar}, T_{\text {in }}=7^{\circ} \mathrm{C}, T_{0}=50^{\circ} \mathrm{C}, p_{0}=0.1 \mathrm{mbar}, X_{0} \approx 0.05 \mathrm{~kg} / \mathrm{kg}$

\section{References}

[1] K. E. N'Tsoukpoe, H. Liu, N. Le Pierres, L. Luo, A review on long-term sorption solar energy storage, Renewable \& Sustainable Energy Reviews 13 (9) (2009) 2385-2396. doi:10.1016/j.rser. 2009.05.008.

[2] H. Schreiber, S. Graf, F. Lanzerath, A. Bardow, Adsorption thermal energy storage for cogeneration in industrial batch processes: Experiment, dynamic modeling and system analysis, Applied Thermal Engineering 89 (2015) 485-493. doi : 10.1016/j . applthermaleng.2015.06.016. URL http://www.sciencedirect.com/science/article/pii/ S1359431115005694

[3] B. Formisano, C. Bonten, Extruded zeolitic honeycombs for sorptive heat storage, AIP Conference Proceedings 1779 (1) (2016) 030003. doi:10. 1063/1.4965473.

[4] J. Jaenchen, T. H. Herzog, K. Gleichmann, B. Unger, A. Brandt, G. Fischer, H. Richter, Performance of an open thermal adsorption storage system with linde type a zeolites: Beads versus honeycombs, Microporous and Mesoporous Materials 207 (2015) 179-184. doi:10.1016/ j.micromeso.2015.01.018.
[5] F. Akhtar, L. Andersson, S. Ogunwumi, N. Hedin, L. Bergström, Structuring adsorbents and catalysts by processing of porous powders, Journal of the European Ceramic Society 34 (7) (2014) 1643-1666. doi: 10.1016/j.jeurceramsoc. 2014.01.008.

[6] H. Liu, K. Nagano, D. Sugiyama, J. Togawa, M. Nakamura, Honeycomb filters made from mesoporous composite material for an open sorption thermal energy storage system to store low-temperature industrial waste heat, International Journal of Heat and Mass Transfer 65 (2013) 471-480. doi:10.1016/j.ijheatmasstransfer.2013.06.021.

[7] M. Schaefer, A. Thess, One-dimensional model of a closed lowpressure adsorber for thermal energy storage, International Journal of Heat and Mass Transfer 117 (Supplement C) (2018) 571-583. doi:10.1016/j.ijheatmasstransfer.2017.09.095.

URL http://www.sciencedirect.com/science/article/pii/ S0017931017314400

[8] B. Mette, H. Kerskes, H. Drück, H. Müller-Steinhagen, Experimental and numerical investigations on the water vapor adsorption isotherms and kinetics of binderless zeolite 13x, International Journal of Heat and Mass Transfer 71 (2014) 555-561. doi: 10.1016/j . ijheatmasstransfer. 2013.12.061.

[9] P. Tatsidjodoung, N. Le Pierrès, J. Heintz, D. Lagre, L. Luo, F. Durier, Experimental and numerical investigations of a zeolite $13 \mathrm{x} /$ water reactor for solar heat storage in buildings, Energy Conversion and Management 108 (2016) 488-500. doi : 10.1016/j .enconman . 2015.11.011.

[10] K. E. N'Tsoukpoe, G. Restuccia, T. Schmidt, X. Py, The size of sorbents in low pressure sorption or thermochemical energy storage processes, Energy 77 (2014) 983-998. doi : 10.1016/j . energy . 2014 .10.013.

[11] L. Yong, K. Sumathy, Review of mathematical investigation on the closed adsorption heat pump and cooling systems, Renewable \& Sustainable Energy Reviews 6 (4) (2002) 305-337. doi:10.1016/S1364-0321(02) 00010-2.

[12] G. G. Ilis, M. Mobedi, S. Ülkü, Comparison of uniform and non-uniform pressure approaches used to analyze an adsorption process in a closed type adsorbent bed, Transport in Porous Media 98 (1) (2013) 81-101. doi:10.1007/s11242-013-0134-1.

URL http: //dx.doi.org/10.1007/s11242-013-0134-1

[13] K. C. Chan, C. Y. H. Chao, G. N. Sze-To, K. S. Hui, Performance predictions for a new zeolite $13 \mathrm{x} / \mathrm{cacl} 2$ composite adsorbent for adsorption cooling systems, International Journal of Heat and Mass Transfer 55 (11-12) (2012) 3214-3224. doi:10.1016/j.ijheatmasstransfer.2012. 02.054.

[14] I. Solmus, D. A. S. Rees, C. Yamali, D. Baker, A two-energy equation model for dynamic heat and mass transfer in an adsorbent bed using silica gel/water pair, International Journal of Heat and Mass Transfer 55 (19-20) (2012) 5275-5288. doi:10.1016/j.ijheatmasstransfer.2012. 05.036.

[15] A. Pesaran, H. Lee, Y. Hwang, R. Radermacher, H.-H. Chun, Review article: Numerical simulation of adsorption heat pumps, Energy 100 (2016) 310-320. doi:10.1016/j.energy .2016.01.103.

[16] M. Duquesne, J. Toutain, A. Sempey, S. Ginestet, Palomo del Barrio, Elena, Modeling of a nonlinear thermochemical energy storage by adsorption on zeolites, Applied Thermal Engineering 71 (1) (2014) 469480. doi:10.1016/j.applthermaleng.2014.07.002.

[17] C. A. Grande, S. Cavenati, P. Barcia, J. Hammer, H. G. Fritz, A. E. RODRIGUES, Adsorption of propane and propylene in zeolite 4a honeycomb monolith, Chemical Engineering Science 61 (10) (2006) 30533067. doi : 10.1016/j.ces. 2005.11.058.

[18] L. Yong, K. Sumathy, Comparison between heat transfer and heat mass transfer models for transportation process in an adsorbent bed, International Journal of Heat and Mass Transfer 47 (8-9) (2004) 1587-1598. doi:10.1016/j.ijheatmasstransfer.2003.10.025.

[19] F. Sharipov, I. Graur, General approach to transient flows of rarefied gases through long capillaries, Vacuum 100 (2014) 22-25. doi:10.1016/j. vacuum. 2013.07.017.

[20] I. Graur, M. T. Ho, Rarefied gas flow through a long rectangular channel of variable cross section, Vacuum 101 (2014) 328-332. doi:10.1016/ j.vacuum. 2013.07.047.

[21] L. F. Shampine, M. W. Reichelt, The matlab ode suite, Siam Journal on Scientific Computing 18 (1) (1997) 1-22. doi:10.1137/ S1064827594276424.

[22] K. Ogata, Y. Yang, Modern control engineering, Vol. 4, Prentice hall In- 
dia, 2002. 